\title{
NITSCHE'S METHOD FOR A TRANSPORT PROBLEM IN TWO-PHASE INCOMPRESSIBLE FLOWS
}

\author{
ARNOLD REUSKEN AND TRUNG HIEU NGUYEN*
}

\begin{abstract}
We consider a parabolic interface problem which models the transport of a dissolved species in two-phase incompressible flow problems. Due to the so-called Henry interface condition the solution is discontinuous across the interface. We use an extended finite element space combined with a method due to Nitsche for the spatial discretization of this problem and derive optimal discretization error bounds for this method. For the time discretization a standard $\theta$-scheme is applied. Results of numerical experiments are given that illustrate the convergence properties of this discretization.
\end{abstract}

Key words. Nitsche's method, interface problem, extended finite elements, two-phase flows,

AMS subject classifications. $65 \mathrm{~N} 30,65 \mathrm{~N} 40$

1. Introduction. Let $\Omega \subset \mathbb{R}^{d}, d=2,3$, be a convex polygonal domain that contains two different immiscible incompressible phases. The (in general time dependent) subdomains containing the two phases are denoted by $\Omega_{1}, \Omega_{2}$, with $\bar{\Omega}=\bar{\Omega}_{1} \cup \bar{\Omega}_{2}$. A typical example is a droplet surrounded by another fluid. In this paper we only consider the stationary case in which the interface $\Gamma:=\bar{\Omega}_{1} \cap \bar{\Omega}_{2}$ does not depend on time. The interface $\Gamma$ is assumed to be sufficiently smooth. A model example is a droplet at a stationary position in a flow field. The fluid dynamics in such a flow problem is usually modeled by the incompressible Navier-Stokes equations combined with suitable conditions at the interface which describe the effect of surface tension. For this model we refer to the literature, e.g. [2, 7, 19, 27]. By w we denote the velocity field resulting from these Navier-Stokes equations. In this paper we consider a model which describes the transport of a dissolved species in such a two-phase flow problem. In strong formulation this model is as follows:

$$
\begin{aligned}
\frac{\partial u}{\partial t}+\mathbf{w} \cdot \nabla u-\operatorname{div}(\alpha \nabla u) & =f \quad \text { in } \Omega_{i}, i=1,2, t \in[0, T], \\
{[\alpha \nabla u \cdot \mathbf{n}]_{\Gamma} } & =0, \\
{[\beta u]_{\Gamma} } & =0, \\
u(\cdot, 0) & =u_{0} \quad \text { in } \Omega_{i}, \quad i=1,2, \\
u(\cdot, t) & =0 \quad \text { on } \partial \Omega, \quad t \in[0, T] .
\end{aligned}
$$

Here $\mathbf{n}$ denotes the unit normal at $\Gamma$ pointing from $\Omega_{1}$ into $\Omega_{2}$. For a sufficiently smooth function $v,[v]=[v]_{\Gamma}$ denotes the jump of $v$ across $\Gamma$, i.e. $[v]=\left(v_{1}\right)_{\mid \Gamma}-$ $\left(v_{2}\right)_{\mid \Gamma}$, where $v_{i}=v_{\mid \Omega_{i}}$ is the restriction of $v$ to $\Omega_{i}$. In (1.1) we have standard parabolic convection-diffusion equations in the two subdomains $\Omega_{1}$ and $\Omega_{2}$. The diffusion coefficient $\alpha$ is assumed to be piecewise constant:

$$
\alpha=\alpha_{i}>0 \text { in } \Omega_{i} .
$$

In general we have $\alpha_{1} \neq \alpha_{2}$. The interface condition in (1.2) results from the conservation of mass principle. The condition in (1.3) is the so-called Henry condition, cf.

\footnotetext{
*Institut für Geometrie und Praktische Mathematik, RWTH-Aachen University, D-52056 Aachen, Germany; email: reusken@igpm.rwth-aachen.de
} 
$[17,23,22,3,2]$. In this condition the coefficient $\beta$ is strictly positive and piecewise constant:

$$
\beta=\beta_{i}>0 \text { in } \Omega_{i} .
$$

In general we have $\beta_{1} \neq \beta_{2}$, since species concentration usually has a jump discontinuity at the interface due to different solubility within the respective fluid phases. Hence, the solution $u$ is discontinuous across the interface.

In this paper we analyze a special finite element method for the discretization of this class of parabolic interface problems. For the special case $\beta_{1}=\beta_{2}$ (no discontinuity) and with a triangulation which is fitted to the interface, standard finite element spaces have (close to) optimal approximation properties. In [4] it is proved that in this special case for standard linear finite elements an $L^{2}$-discretization error bound of the order $h^{2} \log h$ holds.

In this paper we allow $\beta_{1} \neq \beta_{2}$ and use triangulations that are unfitted (as in level set of VOF approaches), i.e. the interface crosses the elements. We will analyze a variant of Nitsche's method [20] for the spatial discretization of this problem. From this semi-discrete problem a full discretization is obtained by using a standard $\theta$ scheme for time discretization. We use the same Nitsche method as presented and analyzed in [8], cf. also $[9,10,11]$. In that paper this method is applied to a stationary heat conduction problem with a conductivity that is discontinuous across the interface $\left(\alpha_{1} \neq \alpha_{2}\right)$ but with a solution that is continuous across the interface $\left(\beta_{1}=\beta_{2}\right)$. We apply this method to the instationary problem described above, with $\beta_{1} \neq \beta_{2}$ (discontinuous solution), and furthermore allow a convection term in (1.1) (in [8] only pure diffusion is considered). In the error analysis that we present some key results from $[8]$ are used.

We also mention the papers $[12,13,14,15,16]$ in which a similar Nitsche method is applied and analyzed in a different setting, namely as a mortar method, which allows the use of non-matching meshes, for the discretization of elliptic and parabolic problems with smooth solutions.

REMARK 1 . The discontinuity of $u$ across the interface can be avoided by introducing transformed quantities $\tilde{u}:=\beta u, \tilde{\alpha}:=\alpha / \beta, \tilde{\mathbf{w}}:=\mathbf{w} / \beta$. Then (1.1)-(1.3) can be reformulated as

$$
\begin{aligned}
\beta^{-1} \frac{\partial \tilde{u}}{\partial t}+\tilde{\mathbf{w}} \cdot \nabla \tilde{u}-\operatorname{div}(\tilde{\alpha} \nabla \tilde{u}) & =f \quad \text { in } \quad \Omega_{i}, i=1,2, t \in[0, T], \\
{[\tilde{\alpha} \nabla \tilde{u} \cdot \mathbf{n}]_{\Gamma} } & =0 \\
{[\tilde{u}]_{\Gamma} } & =0 .
\end{aligned}
$$

In this formulation we have continuity of $\tilde{u}$ across $\Gamma$ but, compared to (1.1), a subdomain dependent scaling factor $\beta^{-1}$ in front of the time derivative.

We will consider the model in the formulation (1.1)-(1.5). The discretization method obtained for this model immediately yields an analogon for the transformed model (1.6)-(1.8), cf. remark 5.

The paper is organized as follows. In section 2 we discuss a weak formulation of the problem (1.1)-(1.5). In section 3 Nitsche's finite element method for the spatial discretization is presented. In section 4 optimal discretization error bounds are derived. In section 5 the issue of time discretization is briefly addressed. Finally, in section 6 we present results of a numerical experiment with a three-dimensional transport problem of the form (1.1)-(1.5). 
2. Weak formulation. In this section we give a weak formulation of the problem (1.1)-(1.5) which, under reasonable assumptions on the data $f, u_{0}$ (and $\mathbf{w}$ ), has a unique solution. We assume that for the function $u_{0}$ in the initial condition (1.4) the conditions in (1.2), (1.3) are satisfied. For simplicity we only consider homogeneous Dirichlet boundary conditions in (1.5). Note that this boundary condition is given (only) on $\partial \Omega$ and thus if $\partial \Omega_{1} \cap \partial \Omega=\emptyset$, then it does not prescribe values for $u_{1}=u_{\mid \Omega_{1}}$.

Due to the fact that the underlying two-phase fluid dynamics concerns two incompressible immiscible phases it is reasonable to make the following assumption about the velocity field $\mathbf{w}$ :

$$
\operatorname{div} \mathbf{w}=0 \quad \text { in } \Omega_{i}, i=1,2, \text { and } \mathbf{w} \cdot \mathbf{n}=0 \quad \text { at } \Gamma, \quad\|\mathbf{w}\|_{L^{\infty}(\Omega)} \leq c<\infty .
$$

In the remainder of the paper we assume that (2.1) holds.

For a weak formulation we introduce suitable Hilbert spaces. We define $H_{0}^{1}\left(\Omega_{1} \cup \Omega_{2}\right):=$ $\left\{v \in L^{2}(\Omega) \mid v_{i} \in H^{1}\left(\Omega_{i}\right), i=1,2, v_{\mid \partial \Omega}=0\right\}$, where $v_{i}:=v_{\mid \Omega_{i}}$, and

$$
H:=L^{2}(\Omega), \quad V:=\left\{v \in H_{0}^{1}\left(\Omega_{1} \cup \Omega_{2}\right) \mid[\beta v]_{\Gamma}=0\right\} .
$$

On $H$ we use the scalar product

$$
(u, v)_{0}:=\int_{\Omega} \beta u v d x
$$

which clearly is equivalent to the standard scalar product on $L^{2}(\Omega)$. The corresponding norm is denoted by $\|\cdot\|_{0}$. For $u, v \in H^{1}\left(\Omega_{i}\right)$ we define $(u, v)_{1, \Omega_{i}}:=\beta_{i} \int_{\Omega_{i}} \nabla u \cdot \nabla v d x$ and furthermore

$$
(u, v)_{1, \Omega_{1} \cup \Omega_{2}}:=(u, v)_{1, \Omega_{1}}+(u, v)_{1, \Omega_{2}}=\sum_{j=1}^{2}\left(\frac{\partial u}{\partial x_{j}}, \frac{\partial v}{\partial x_{j}}\right)_{0}, \quad u, v \in V .
$$

The corresponding norm is denoted by $|\cdot|_{1, \Omega_{1} \cup \Omega_{2}}$. This norm is equivalent with

$$
\left(\|\cdot\|_{0}^{2}+|\cdot|_{1, \Omega_{1} \cup \Omega_{2}}^{2}\right)^{\frac{1}{2}}=:\|\cdot\|_{1, \Omega_{1} \cup \Omega_{2}} .
$$

The space $\left(V,(\cdot, \cdot)_{1, \Omega_{1} \cup \Omega_{2}}\right)$ is a Hilbert space. We obtain a Gelfand triple $V \hookrightarrow H \equiv$ $H^{\prime} \hookrightarrow V^{\prime}$, with dense and continuous embeddings $\hookrightarrow$.

We now introduce the bilinear form

$$
a(u, v):=(\alpha u, v)_{1, \Omega_{1} \cup \Omega_{2}}+(\mathbf{w} \cdot \nabla u, v)_{0}, \quad u, v \in V .
$$

This bilinear form is continuous on $V$ and using (2.1) we get, for $u \in V$,

$$
\begin{aligned}
(\mathbf{w} \cdot \nabla u, u)_{0} & =\sum_{i=1,2} \beta_{i} \int_{\Omega_{i}} \mathbf{w} \cdot \nabla u_{i} u_{i} d x \\
& =\int_{\Gamma} \mathbf{w} \cdot \mathbf{n}\left[\beta u^{2}\right]_{\mid \Gamma} d s-\sum_{i=1,2} \beta_{i} \int_{\Omega_{i}} \operatorname{div} \mathbf{w} u_{i}^{2} d x-(\mathbf{w} \cdot \nabla u, u)_{0} \\
& =-(\mathbf{w} \cdot \nabla u, u)_{0} .
\end{aligned}
$$

Hence, $(\mathbf{w} \cdot \nabla u, u)_{0}=0$ holds. This yields ellipticity of $a(\cdot, \cdot)$ :

$$
a(u, u) \geq\left(\min _{i=1,2} \alpha_{i}\right)|u|_{1, \Omega_{1} \cup \Omega_{2}}^{2} \text { for all } u \in V .
$$


We introduce some further standard notation. If $X$ is a Banach space then $L^{2}(0, T ; X)$ is the space of $L^{2}$ functions from $(0, T)$ into $X$, which is a Banach space for the norm

$$
\|f\|_{L^{2}(0, T ; X)}=\left(\int_{0}^{T}\|f(t)\|_{X}^{2} d t\right)^{\frac{1}{2}} .
$$

Furthermore $C([0, T] ; X)$ denotes the space of continuous functions from $[0, T]$ into $X$, which is a Banach space for the norm

$$
\|f\|_{C([0, T] ; X)}=\sup _{t \in[0, T]}\|f(t)\|_{X} .
$$

Now consider the following weak formulation of (1.1)-(1.5). Given $f \in V^{\prime}, u_{0} \in H$, determine $u \in L^{2}(0, T ; V)$ such that

$$
u(0)=u_{0}, \quad\left\langle\frac{\partial u}{\partial t}, u\right\rangle+a(u, v)=\langle f, v\rangle \quad \text { for all } v \in V .
$$

Here $\langle\cdot, \cdot\rangle$ denotes the duality pairing on $V^{\prime} \times V$. The derivative $\frac{\partial u}{\partial t}$ is defined in a distributional sense, cf. for example [18, 25]. In particular $\frac{\partial u}{\partial t} \in L^{2}\left(0, T ; V^{\prime}\right)$. It can be shown $([18,25])$ that $u \in C([0, T] ; H)$ holds and thus the initial condition $u=u_{0}$ is well-defined. It is proved in $[18,25]$ that the weak formulation $(2.4)$ has a unique solution.

REMARK 2. This existence and uniqueness result still holds (cf. $[26,6])$ if instead of ellipticity of the bilinear form $a(\cdot, \cdot)$, cf. (2.3), one has the weaker property

$$
a(u, u) \geq c_{0}|u|_{1, \Omega_{1} \cup \Omega_{2}}^{2}-c_{1}\|u\|_{0}^{2} \text { for all } u \in V,
$$

with constants $c_{0}>0$ and $c_{1}$ independent of $u$. Using $\left|(\mathbf{w} \cdot \nabla u, u)_{0}\right| \leq c|u|_{1, \Omega_{1} \cup \Omega_{2}}\|u\|_{0}$ it easily follows that this property holds without using the first two assumptions in (2.1). We introduce these assumptions because they simplify the presentation of the analysis for the continuous problem and we need them in our analysis of Nitsche's method in section 4 .

The duality pairing in (2.4) can be replaced by the scalar product $(\cdot, \cdot)_{0}$ on $H$ if one assumes additional regularity of the data $f$ and $u_{0}$. Related to this regularity issue we first consider the stationary problem: for $f \in H$,

$$
\text { find } w \in V \text { such that } a(w, v)=(f, v)_{0} \text { for all } v \in V \text {. }
$$

The unique solution $w$ of this problem satisfies (cf. [4])

$$
w \in V_{\text {reg }}:=\left\{v \in V \mid v_{i} \in H^{2}\left(\Omega_{i}\right), \quad i=1,2 .\right\},
$$

and

$$
\|w\|_{2, \Omega_{1} \cup \Omega_{2}}:=\left(\|w\|_{1, \Omega_{1} \cup \Omega_{2}}^{2}+|w|_{2, \Omega_{1} \cup \Omega_{2}}^{2}\right)^{\frac{1}{2}} \leq c\|f\|_{0}
$$

holds, with a constant $c$ independent of $f$. The space $V_{\text {reg }}$ is a Banach space with respect to the norm $\|\cdot\|_{2, \Omega_{1} \cup \Omega_{2}}$. Using this regularity result it follows from Theorem 3.2 in [24] that the following holds:

Lemma 2.1. Take $f \in H, u_{0} \in V_{\text {reg }}$ There exists a unique $u \in C\left([0, T] ; V_{\text {reg }}\right)$ such that $u(0)=u_{0}$ and

$$
\left(\frac{\partial u}{\partial t}, v\right)_{0}+a(u, v)=(f, v)_{0} \quad \text { for all } \quad v \in V .
$$


Moreover, the distributional time derivative satisfies

$$
\frac{\partial u}{\partial t} \in L^{2}(0, T ; V) \cap C([0, T] ; H) .
$$

We now show that the variational problem (2.8) is indeed a correct weak formulation of the problem (1.1)-(1.5).

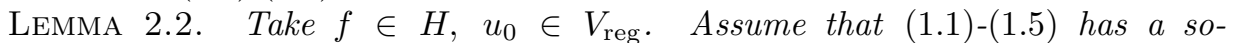
lution $u(x, t)$ which is sufficiently smooth such that for $u: t \rightarrow u(\cdot, t)$ we have $u \in C\left([0, T] ; V_{\mathrm{reg}}\right)$ and $\frac{\partial u}{\partial t} \in L^{2}(0, T ; H)$. This u solves the variational problem (2.8). Conversely, if $u \in C\left([0, T] ; V_{\mathrm{reg}}\right)$ with $u(0)=u_{0}$ solves the variational problem (2.8) then $u$ satisfies (1.1) in a weak $L^{2}\left(\Omega_{i}\right)$ sense and (1.2), (1.3), (1.5) in trace sense. we get

Proof. Take $u \in C\left([0, T] ; V_{\text {reg }}\right)$ with $\frac{\partial u}{\partial t} \in L^{2}(0, T ; H)$, and $v \in V$. Using $[\beta v]=0$

$$
\begin{aligned}
{[\alpha \nabla u \cdot \mathbf{n} \beta v]_{\Gamma}=} & {[\alpha \nabla u \cdot \mathbf{n}]_{\Gamma} \frac{1}{2}\left(\left(\beta_{1} v_{1}\right)_{\mid \Gamma}+\left(\beta_{2} v_{2}\right)_{\mid \Gamma}\right) } \\
& +\frac{1}{2}\left(\left(\alpha_{1} \nabla u_{1} \cdot \mathbf{n}\right)_{\mid \Gamma}+\left(\alpha_{2} \nabla u_{2} \cdot \mathbf{n}\right)_{\mid \Gamma}\right)[\beta v]_{\mid \Gamma} \\
= & {[\alpha \nabla u \cdot \mathbf{n}]_{\Gamma} \frac{1}{2}\left(\left(\beta_{1} v_{1}\right)_{\mid \Gamma}+\left(\beta_{2} v_{2}\right)_{\mid \Gamma}\right) . }
\end{aligned}
$$

Using this we obtain

$$
\begin{aligned}
& \left(\frac{\partial u}{\partial t}, v\right)_{0}+a(u, v) \\
& =\left(\frac{\partial u}{\partial t}, v\right)_{0}+(\mathbf{w} \cdot \nabla u, v)_{0}-\sum_{i=1,2} \int_{\Omega_{i}} \operatorname{div}\left(\alpha_{i} \nabla u\right) \beta_{i} v d x+\int_{\Gamma}[\alpha \nabla u \cdot \mathbf{n} \beta v]_{\Gamma} d s \\
& =\sum_{i=1,2} \int_{\Omega_{i}}\left(\frac{\partial u}{\partial t}+\mathbf{w} \cdot \nabla u-\operatorname{div}\left(\alpha_{i} \nabla u\right)\right) \beta_{i} v d x \\
& \quad+\int_{\Gamma}[\alpha \nabla u \cdot \mathbf{n}]_{\Gamma} \frac{1}{2}\left(\left(\beta_{1} v_{1}\right)_{\mid \Gamma}+\left(\beta_{2} v_{2}\right)_{\mid \Gamma}\right) d s .
\end{aligned}
$$

If $u$ satisfies (1.1), (1.2) we thus obtain

$$
\left(\frac{\partial u}{\partial t}, v\right)_{0}+a(u, v)=(f, v)_{0} \quad \text { for all } v \in V,
$$

i.e., (2.8) holds. Conversely, if $u \in C\left([0, T] ; V_{\text {reg }}\right)$ with $u(0)=u_{0}$ solves the variational problem (2.8) we obtain

$$
\begin{gathered}
\sum_{i=1,2} \int_{\Omega_{i}}\left(\frac{\partial u}{\partial t}+\mathbf{w} \cdot \nabla u-\operatorname{div}\left(\alpha_{i} \nabla u\right)-f\right) \beta_{i} v d x \\
+\int_{\Gamma}[\alpha \nabla u \cdot \mathbf{n}]_{\Gamma} \frac{1}{2}\left(\left(\beta_{1} v_{1}\right)_{\mid \Gamma}+\left(\beta_{2} v_{2}\right)_{\mid \Gamma}\right) d s=0
\end{gathered}
$$

for all $v \in V$. This implies that $\frac{\partial u}{\partial t}+\mathbf{w} \cdot \nabla u-\operatorname{div}\left(\alpha_{i} \nabla u\right)=f$ in $L^{2}\left(\Omega_{i}\right)$ sense and $[\alpha \nabla u \cdot \mathbf{n}]_{\Gamma}=0$ in trace sense. The properties in (1.3) and (1.5) hold due to $u \in V$.

For the result in (2.10) it is essential that we multiply the equation (1.1) by $\beta v$ and not by $v$. This explains why in the scalar products $(\cdot, \cdot)_{0}$ and $(\cdot, \cdot)_{1, \Omega_{1} \cup \Omega_{2}}$ we use the weighting with the (piecewise constant) function $\beta$. 
3. Nitsche's method. We present Nitsche's method along the same lines as in [8]. Let $\left\{\mathcal{T}_{h}\right\}_{h>0}$ be a family of shape regular triangulations of $\Omega$. A triangulation $\mathcal{T}_{h}$ consists of triangles $T$, with $h_{T}:=\operatorname{diam}(T)$ and $h:=\max \left\{h_{T} \mid T \in \mathcal{T}_{h}\right\}$. For any triangle $T \in \mathcal{T}_{h}$ let $T_{i}:=T \cap \Omega_{i}$ be the part of $T$ in $\Omega_{i}$. For any $T$ with $T \cap \Gamma \neq \emptyset$ we define $\Gamma_{T}:=T \cap \Gamma$. Related to the triangulation we formulate the same assumptions as in [8]:

Assumption 1. Consider a $T$ with $T \cap \Gamma \neq \emptyset$. We assume that the interface $\Gamma$ intersects $\partial T$ exactly twice and each edge of $T$ at most once. Let $\Gamma_{T, h}$ be the straight line connecting the points of intersection between $\Gamma$ and $\partial T$. We assume that $\Gamma_{T}$ is a function of length on $\Gamma_{T, h}$ :

$$
\Gamma_{T, h}=\left\{(\xi, \eta)|0<\xi<| \Gamma_{T, h} \mid, \eta=0\right\}, \quad \Gamma_{T}=\left\{(\xi, \eta)|0<\xi<| \Gamma_{T, h} \mid, \eta=\delta(\xi)\right\} .
$$

The assumptions formulated in assumption 1 are satisfied on sufficiently fine meshes. We now introduce the finite element space

$$
V_{h}^{\Gamma}:=\left\{v \in H_{0}^{1}\left(\Omega_{1} \cup \Omega_{2}\right) \mid v_{\mid T_{i}} \text { is linear for all } T \in \mathcal{T}_{h}, i=1,2 .\right\}
$$

Note that $V_{h}^{\Gamma} \subset H_{0}^{1}\left(\Omega_{1} \cup \Omega_{2}\right)$, but $V_{h}^{\Gamma} \not \subset V$, since the Henry interface condition $\left[\beta v_{h}\right]=0$ does not necessarily hold for $v_{h} \in V_{h}^{\Gamma}$.

REMARK 3. In the literature a finite element discretization based on the space $V_{h}^{\Gamma}$ is often called an extended finite element method (XFEM), cf. [1, 5]. Furthermore, in the (engineering) literature this space is usually characterized in a different way, which we briefly explain. Let $V_{h}$ be the standard finite element space of continuous piecewise linears, corresponding to the triangulation $\mathcal{T}_{h}$. Define the index set $\mathcal{J}=\{1, \ldots, n\}$, where $n=\operatorname{dim} V_{h}$, and let $\left(\phi_{i}\right)_{i \in \mathcal{J}}$ be the nodal basis in $V_{h}$. Let $\mathcal{J}_{\Gamma}:=\{j \in$ $\left.\mathcal{J}|| \Gamma \cap \operatorname{supp}\left(\phi_{j}\right) \mid>0\right\}$ be the index set of those basis functions the support of which is intersected by $\Gamma$. The heaviside function $H_{\Gamma}$ has the values $H_{\Gamma}(x)=0$ for $x \in \Omega_{1}$, $H_{\Gamma}(x)=1$ for $x \in \Omega_{2}$. Using this, for $j \in \mathcal{J}_{\Gamma}$ we introduce a so-called enrichment function $\Phi_{j}(x):=H_{\Gamma}(x)-H_{\Gamma}\left(x_{j}\right)$, where $x_{j}$ is the vertex with index $j$. We introduce a new basis function $\phi_{j}^{\Gamma}:=\phi_{j} \Phi_{j}, j \in \mathcal{J}_{\Gamma}$, and define the space

$$
V_{h}^{\Gamma}:=V_{h} \oplus \operatorname{span}\left\{\phi_{j}^{\Gamma} \mid j \in \mathcal{J}_{\Gamma}\right\} .
$$

This characterization accounts for the name "extended finite element method". The new basis functions $\phi_{j}^{\Gamma}$ have the property $\phi_{j}^{\Gamma}\left(x_{i}\right)=0$ for all $i \in \mathcal{J}$. An $L^{2}$-stability property of the basis $\left(\phi_{j}\right)_{j \in \mathcal{J}} \cup\left(\phi_{j}^{\Gamma}\right)_{j \in J_{\Gamma}}$ of $V_{h}^{\Gamma}$ is given in [21].

Define

$$
\left(\kappa_{i}\right)_{\mid T}=\frac{\left|T_{i}\right|}{|T|}, \quad T \in \mathcal{T}_{h}, i=1,2,
$$

hence, $\kappa_{1}+\kappa_{2}=1$. For $v$ sufficiently smooth such that $\left(v_{i}\right)_{\mid \Gamma}, i=1,2$, are well-defined, we define the weighted average

$$
\{v\}:=\kappa_{1}\left(v_{1}\right)_{\mid \Gamma}+\kappa_{2}\left(v_{2}\right)_{\mid \Gamma} .
$$

For the average and jump operators the following identity holds for all $f, g$ such that these operators are well-defined:

$$
[f g]=\{f\}[g]+[f]\{g\}-\left(\kappa_{1}-\kappa_{2}\right)[f][g] .
$$


Let $(f, g)_{\Gamma}:=\int_{\Gamma} f g d s$ be the $L^{2}(\Gamma)$ scalar product. We introduce the bilinear form

$$
\begin{aligned}
a_{h}(u, v): & =(\alpha u, v)_{1, \Omega_{1} \cup \Omega_{2}}+(\mathbf{w} \cdot \nabla u, v)_{0}-([\beta u],\{\alpha \nabla v \cdot \mathbf{n}\})_{\Gamma} \\
& -(\{\alpha \nabla u \cdot \mathbf{n}\},[\beta v])_{\Gamma}+\lambda h^{-1}([\beta u],[\beta v])_{\Gamma},
\end{aligned}
$$

with $\lambda>0$ a parameter. This bilinear form is well-defined on the space $V_{h}^{\Gamma}$ but also on

$$
W_{\text {reg }}:=\left\{v \in H_{0}^{1}\left(\Omega_{1} \cup \Omega_{2}\right) \mid v_{i} \in H^{2}\left(\Omega_{i}\right), i=1,2 .\right\} .
$$

The space $W_{\text {reg }}$ is larger than the space $V_{\text {reg }}$ in (2.6). The interface condition $[\beta v]$ is

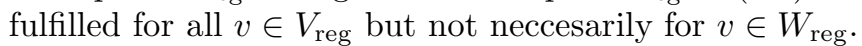

Using this bilinear form we define a method of lines discretization of (2.8). Let $\hat{u}_{0} \in V_{h}^{\Gamma}$ be an approximation of $u_{0}$. For $t \in[0, T]$ let $u_{h}(t) \in V_{h}^{\Gamma}$ be such that $u_{h}(0)=\hat{u}_{0}$ and

$$
\left(\frac{\partial u_{h}}{\partial t}, v_{h}\right)_{0}+a_{h}\left(u_{h}, v_{h}\right)=\left(f, v_{h}\right)_{0} \text { for all } v_{h} \in V_{h}^{\Gamma} .
$$

Opposite to the weak formulation in (2.8), in this discretization method the Henry interface condition $\left[\beta u_{h}\right]=0$ is not treated as an "essential" interface condition in the finite element space $V_{h}^{\Gamma}$. This interface condition is satisfied only approximately by introducing the (penalty) term $\lambda h^{-1}([\beta u],[\beta v])_{\Gamma}$ in the bilinear form $a_{h}(\cdot, \cdot)$. As we will show in the following sections, this approach leads to optimal order error bounds (section 4) and satisfactory results in numerical experiments (section 6).

4. Analysis of Nitsche's method. In this section we present an error analysis of the method of lines discretization given in (3.4). We start with a consistency result:

Lemma 4.1. Let $u=u(t) \in V_{\text {reg }}$ be the solution defined in lemma 2.1. Then $u(t)$ satisfies

$$
\left(\frac{\partial u}{\partial t}, v_{h}\right)_{0}+a_{h}\left(u, v_{h}\right)=\left(f, v_{h}\right) \quad \text { for al } v_{h} \in V_{h}^{\Gamma}, \quad t \in[0, T] .
$$

Proof. From lemma 2.2 we have that $u=u(t)$ satisfies $[\alpha \nabla u \cdot \mathbf{n}]=0,[\beta u]=0$. Using this and (3.2) we obtain:

$$
\begin{aligned}
& -\sum_{i=1,2} \int_{\Omega_{i}} \operatorname{div}\left(\alpha_{i} \nabla u\right) \beta v_{h} d x+\left(\mathbf{w} \cdot \nabla u, v_{h}\right)_{0} \\
& =-\int_{\Gamma}\left[\alpha \nabla u \cdot \mathbf{n} \beta v_{h}\right] d s+\left(\alpha u, v_{h}\right)_{1, \Omega_{1} \cup \Omega_{2}}+\left(\mathbf{w} \cdot \nabla u, v_{h}\right)_{0} \\
& =-\left(\{\alpha \nabla u \cdot \mathbf{n}\},\left[\beta v_{h}\right]\right)_{\Gamma}+\left(\alpha u, v_{h}\right)_{1, \Omega_{1} \cup \Omega_{2}}+\left(\mathbf{w} \cdot \nabla u, v_{h}\right)_{0}=a_{h}\left(u, v_{h}\right) .
\end{aligned}
$$

Furthermore, $u$ solves (1.1) (in the sense as in lemma 2.2). Multiplication of (1.1) by $\beta v_{h}$ and integration over $\Omega$ results in

$$
\begin{aligned}
\left(f, v_{h}\right)_{0} & =\left(\frac{\partial u}{\partial t}, v_{h}\right)_{0}+\left(\mathbf{w} \cdot \nabla u, v_{h}\right)_{0}-\sum_{i=1,2} \int_{\Omega_{i}} \operatorname{div}\left(\alpha_{i} \nabla u\right) \beta v_{h} d x \\
& =\left(\frac{\partial u}{\partial t}, v_{h}\right)_{0}+a_{h}\left(u, v_{h}\right),
\end{aligned}
$$


and thus the consistency result holds.

For the error analysis we introduce a suitable norm, as in [8]. Let $G_{h}$ denote the set of all triangles that are intersected by $\Gamma$. We define

$$
\begin{aligned}
\|v\|_{1 / 2, h, \Gamma}^{2} & :=\sum_{T \in G_{h}} h_{T}^{-1}\|v\|_{L^{2}\left(\Gamma_{T}\right)}^{2}, \\
\|v\|_{-1 / 2, h, \Gamma}^{2} & :=\sum_{T \in G_{h}} h_{T}\|v\|_{L^{2}\left(\Gamma_{T}\right)}^{2}, \\
\|v\|^{2} & :=|v|_{1, \Omega_{1} \cup \Omega_{2}}^{2}+\|\{\nabla v \cdot \mathbf{n}\}\|_{-1 / 2, h, \Gamma}^{2}+\|[\beta v]\|_{1 / 2, h, \Gamma}^{2} .
\end{aligned}
$$

Note that different from [8] we have a scaling with $\beta$ in the terms $|v|_{1, \Omega_{1} \cup \Omega_{2}}$ and $\|[\beta v]\|_{1 / 2, h, \Gamma}$. The bilinear form $a_{h}(\cdot, \cdot)$ has the following continuity and ellipticity properties with respect to the norm $\|\cdot\|$.

LEMma 4.2. There exist constants $c_{1}, c_{2}>0$ such that for $\lambda$ sufficiently large (independent of $h$ ) the following holds:

$$
\begin{aligned}
\left|a_{h}(u, v)\right| & \leq c_{1}\|u\|\|v\| \quad \text { for all } u, v \in V_{h}^{\Gamma}+W_{\text {reg }}, \\
a_{h}\left(v_{h}, v_{h}\right) & \geq c_{2}\left\|v_{h}\right\|^{2} \quad \text { for all } v_{h} \in V_{h}^{\Gamma} .
\end{aligned}
$$

Proof. First note that $\left|(f, g)_{\Gamma}\right| \leq\|f\|_{1 / 2, h, \Gamma}\|g\|_{-1 / 2, h, \Gamma}$ holds. Take $u, v \in V_{h}^{\Gamma}+$ $W_{\text {reg }}$. Using the Cauchy-Schwarz inequality and the definitions of the norms we obtain

$$
\begin{aligned}
\left|a_{h}(u, v)\right| \leq & c|u|_{1, \Omega_{1} \cup \Omega_{2}}|v|_{1, \Omega_{1} \cup \Omega_{2}}+c|u|_{1, \Omega_{1} \cup \Omega_{2}}\|v\|_{0} \\
& +\|[\beta u]\|_{1 / 2, h, \Gamma}\|\{\alpha \nabla v \cdot \mathbf{n}\}\|_{-1 / 2, h, \Gamma}+\|\{\alpha \nabla u \cdot \mathbf{n}\}\|_{-1 / 2, h, \Gamma}\|[\beta v]\|_{1 / 2, h, \Gamma} \\
& +\lambda\|[\beta u]\|_{1 / 2, h, \Gamma}\|[\beta v]\|_{1 / 2, h, \Gamma} \leq c\|u\|\|v\|,
\end{aligned}
$$

which proves the continuity. Using the assumptions (2.1) we obtain for $v_{h} \in V_{h}^{\Gamma}$, cf. $(2.2),\left(\mathbf{w} \cdot \nabla v_{h}, v_{h}\right)_{0}=0$. Hence,

$$
\begin{aligned}
a_{h}\left(v_{h}, v_{h}\right) & \geq\left|\alpha^{\frac{1}{2}} v_{h}\right|_{1, \Omega_{1} \cup \Omega_{2}}^{2}-2\left|\left(\left\{\alpha \nabla v_{h} \cdot \mathbf{n}\right\},\left[\beta v_{h}\right],\right)_{\Gamma}\right|+\lambda c\left\|\left[\beta v_{h}\right]\right\|_{1 / 2, h, \Gamma}^{2} \\
& \geq\left|\alpha^{\frac{1}{2}} v_{h}\right|_{1, \Omega_{1} \cup \Omega_{2}}^{2}-2\left\|\left\{\alpha \nabla v_{h} \cdot \mathbf{n}\right\}\right\|_{-1 / 2, h, \Gamma}\|[\beta v]\|_{1 / 2, h, \Gamma}+\lambda c\left\|\left[\beta v_{h}\right]\right\|_{1 / 2, h, \Gamma}^{2},
\end{aligned}
$$

with $c>0$ independent of $h$. From Lemma 4 in [8] we have

$$
\left\|\left\{\alpha \nabla v_{h} \cdot \mathbf{n}\right\}\right\|_{-1 / 2, h, \Gamma} \leq c\left|\alpha^{\frac{1}{2}} v_{h}\right|_{1, \Omega_{1} \cup \Omega_{2}} .
$$

Using this we obtain the ellipticity result in (4.6), provided the parameter $\lambda$ is chosen sufficiently large.

In [8] an interpolation operator $I_{h}^{*}: H_{0}^{1}(\Omega) \cap H^{2}\left(\Omega_{1} \cup \Omega_{2}\right) \rightarrow V_{h}^{\Gamma}$ is defined and an interpolation error bound is proved. This interpolation operator is defined by nodal interpolation of $H^{2}$-extensions of $v_{i}, i=1,2$. The definition and the analysis of this operator does not use the fact that $v \in H_{0}^{1}(\Omega) \cap H^{2}\left(\Omega_{1} \cup \Omega_{2}\right)$ is continuous across $\Gamma$. The definition of $I_{h}^{*}$ and its analysis apply, with only minor changes, to $v \in W_{\text {reg }}$. Furthermore, the analysis of the interpolation error in [8] also applies if in the norm \|\|$\|$ we use a scaling with $\beta$, cf. (4.4). Thus Theorem 2 in [8] yields the following.

THEOREM 4.3. Let $I_{h}^{*}: W_{\text {reg }} \rightarrow V_{h}^{\Gamma}$ be the interpolation operator defined in [8]. There exists a constant $c$ such that

$$
\left\|v-I_{h}^{*} v\right\| \leq c h\|v\|_{2, \Omega_{1} \cup \Omega_{2}} \quad \text { for all } v \in W_{\text {reg }}
$$


holds.

In the error analysis we use the elliptic projection $R_{h}: W_{\text {reg }}+V_{h}^{\Gamma} \rightarrow V_{h}^{\Gamma}$, defined by

$$
a_{h}\left(R_{h} v, w_{h}\right)=a_{h}\left(v, w_{h}\right) \text { for all } w_{h} \in V_{h}^{\Gamma} .
$$

In the following two lemmas we derive error bounds for this projection.

Lemma 4.4. The following holds:

$$
\left\|R_{h} v-v\right\| \leq c h\|v\|_{2, \Omega_{1} \cup \Omega_{2}} \quad \text { for all } v \in W_{\text {reg. }} .
$$

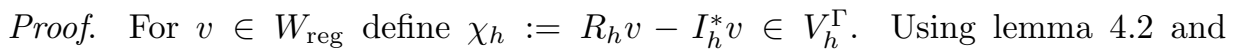
theorem 4.3 we get, with $c_{2}>0$ :

$$
\begin{aligned}
c_{2}\left\|\chi_{h}\right\|^{2} & \leq a_{h}\left(\chi_{h}, \chi_{h}\right)=a_{h}\left(R_{h} v-I_{h}^{*} v, \chi_{h}\right) \\
& =a_{h}\left(v-I_{h}^{*} v, \chi_{h}\right) \leq c_{1}\left\|v-I_{h}^{*} v\right\|\left\|\chi_{h}\right\| \leq c h\|v\|_{2, \Omega_{1} \cup \Omega_{2}}\left\|\chi_{h}\right\| .
\end{aligned}
$$

Hence, $\left\|\chi_{h}\right\| \leq c h\|v\|_{2, \Omega_{1} \cup \Omega_{2}}$ holds and thus

$$
\left\|R_{h} v-v\right\| \leq\left\|\chi_{h}\right\|+\left\|v-I_{h}^{*} v\right\| \leq c h\|v\|_{2, \Omega_{1} \cup \Omega_{2}}
$$

holds.

Lemma 4.5. The following holds:

$$
\left\|R_{h} v-v\right\|_{0} \leq c h^{2}\|v\|_{2, \Omega_{1} \cup \Omega_{2}} \quad \text { for all } v \in W_{\text {reg. }} .
$$

Proof. For $v \in W_{\text {reg }}$ define $e_{h}:=R_{h} v-v \in V_{h}^{\Gamma}+W_{\text {reg. }}$. Introduce the bilinear form

$$
\tilde{a}(u, v)=(\alpha u, v)_{1, \Omega_{1} \cup \Omega_{2}}-(\mathbf{w} \cdot \nabla u, v)_{0}, \quad u, v \in H_{0}^{1}\left(\Omega_{1} \cup \Omega_{2}\right) .
$$

Using $\mathbf{w} \cdot \mathbf{n}=0$ on $\Gamma$ and $\operatorname{div} \mathbf{w}=0$ in $\Omega_{i}$ we get $-(\mathbf{w} \cdot \nabla u, v)_{0}=(\mathbf{w} \cdot \nabla v, u)_{0}$ and thus $\tilde{a}(u, v)=a(v, u)$ for $u, v \in H_{0}^{1}\left(\Omega_{1} \cup \Omega_{2}\right)$. Let $\tilde{u} \in V$ be the unique solution of

$$
\tilde{a}(\tilde{u}, v)=\left(e_{h}, v\right)_{0} \quad \text { for all } v \in V .
$$

This dual problem has the same regularity properties as the one in (2.5), i.e., $\tilde{u} \in$ $H^{2}\left(\Omega_{1} \cup \Omega_{2}\right)$ and

$$
\|\tilde{u}\|_{2, \Omega_{1} \cup \Omega_{2}} \leq c\left\|e_{h}\right\|_{0},
$$

with a constant $c$ independent of $e_{h}$. Using this regularity property, combined with $[\beta \tilde{u}]=0$ (since $\tilde{u} \in V$ ) it follows that $\tilde{u}$ solves the following problem:

$$
\begin{aligned}
-\operatorname{div}(\alpha \nabla \tilde{u})-\mathbf{w} \cdot \nabla \tilde{u} & =e_{h} \quad \text { in } \Omega_{i}, i=1,2, \quad \text { (in } L^{2} \text { sense) }, \\
{[\alpha \nabla \tilde{u} \cdot \mathbf{n}]_{\Gamma} } & =0 \\
{[\beta \tilde{u}]_{\Gamma} } & =0 .
\end{aligned}
$$


Multiplication of (4.8) with $\beta e_{h}$, integration over $\Omega_{i}$ and applying partial integration we obtain, using (4.9),(4.10):

$$
\begin{aligned}
\left(e_{h}, e_{h}\right)_{0}= & \left(\alpha \tilde{u}, e_{h}\right)_{1, \Omega_{1} \cup \Omega_{2}}-\left(\mathbf{w} \cdot \nabla \tilde{u}, e_{h}\right)_{0}-\int_{\Gamma}\left[\alpha \nabla \tilde{u} \cdot \mathbf{n} \beta e_{h}\right] d s \\
= & \left(\alpha e_{h}, \tilde{u}\right)_{1, \Omega_{1} \cup \Omega_{2}}+\left(\mathbf{w} \cdot \nabla e_{h}, \tilde{u}\right)_{0}-\left(\left[\beta e_{h}\right],\{\alpha \nabla \tilde{u} \cdot \mathbf{n}\}\right)_{\Gamma} \\
& -\left(\left\{\alpha \nabla e_{h} \cdot \mathbf{n}\right\},[\beta \tilde{u}]\right)_{\Gamma}+\lambda h^{-1}\left(\left[\beta e_{h}\right],[\beta \tilde{u}]\right)_{\Gamma} \\
= & a_{h}\left(e_{h}, \tilde{u}\right) .
\end{aligned}
$$

Using this in combination with theorem 4.3 and lemma 4.4 we get

$$
\begin{aligned}
\left(e_{h}, e_{h}\right)_{0} & =a_{h}\left(e_{h}, \tilde{u}\right)=a_{h}\left(e_{h}, \tilde{u}-I_{h}^{*} \tilde{u}\right) \leq c_{1}\left\|e_{h}\right\|\left\|\tilde{u}-I_{h}^{*} \tilde{u}\right\| \\
& \leq c h^{2}\|v\|_{2, \Omega_{1} \cup \Omega_{2}}\|\tilde{u}\|_{2, \Omega_{1} \cup \Omega_{2}} \leq c h^{2}\|v\|_{2, \Omega_{1} \cup \Omega_{2}}\left\|e_{h}\right\|_{0},
\end{aligned}
$$

which completes the proof.

We now derive an error bound for the semi-discretization by Nitsche's method in (3.4). We require that the solution $u=u(t) \in V_{\text {reg }}$ as defined in lemma 2.1 has sufficient regularity, in particular $\frac{\partial u}{\partial t} \in L^{1}\left(0, T ; W_{\text {reg }}\right)$. The analysis uses standard arguments as in, for example, [26].

TheOrem 4.6. Let $u=u(t) \in V_{\text {reg }}$ be the solution defined in lemma 2.1 and $u_{h}=u_{h}(t) \in V_{h}^{\Gamma}$ the solution of (3.4) with $u_{h}(0)=\hat{u}_{0}$. The following holds

$\left\|u_{h}(t)-u(t)\right\|_{0} \leq\left\|\hat{u}_{0}-R_{h} u_{0}\right\|_{0}+c h^{2}\left\{\left\|u_{0}\right\|_{2, \Omega_{1} \cup \Omega_{2}}+\int_{0}^{t}\left\|\frac{\partial u}{\partial t}\right\|_{2, \Omega_{1} \cup \Omega_{2}} d \tau\right\}, \quad 0 \leq t \leq T$.

Proof. Introduce the splitting $u_{h}(t)-u(t)=\theta(t)+\rho(t)$, with $\theta:=u_{h}-R_{h} u$, $\rho:=R_{h} u-u$. From lemma 4.5 we have

$$
\|\rho(t)\|_{0} \leq c h^{2}\|u(t)\|_{2, \Omega_{1} \cup \Omega_{2}} \leq c h^{2}\left(\left\|u_{0}\right\|_{2, \Omega_{1} \cup \Omega_{2}}+\int_{0}^{t}\left\|\frac{\partial u}{\partial t}\right\|_{2, \Omega_{1} \cup \Omega_{2}} d \tau\right) .
$$

For $\theta=\theta(t) \in V_{h}^{\Gamma}$ we have, using lemma 4.1:

$$
\begin{aligned}
\|\theta\|_{0} \frac{d}{d t}\|\theta\|_{0} & =\frac{1}{2} \frac{d}{d t}\|\theta\|_{0}^{2}=\left(\frac{\partial \theta}{\partial t}, \theta\right)_{0} \leq\left(\frac{\partial \theta}{\partial t}, \theta\right)_{0}+a_{h}(\theta, \theta) \\
& =\left(\frac{\partial u_{h}}{\partial t}, \theta\right)_{0}+a_{h}\left(u_{h}, \theta\right)-\left(\frac{\partial R_{h} u}{\partial t}, \theta\right)_{0}-a_{h}\left(R_{h} u, \theta\right) \\
& =(f, \theta)_{0}-a_{h}(u, \theta)-\left(\frac{\partial R_{h} u}{\partial t}, \theta\right)_{0} \\
& =\left(\frac{\partial u}{\partial t}, \theta\right)_{0}-\left(\frac{\partial R_{h} u}{\partial t}, \theta\right)_{0}=\left(w-R_{h} w, \theta\right)_{0},
\end{aligned}
$$

with $w=\frac{\partial u}{\partial t}$. We assumed sufficient regularity, in particular $w \in W_{\text {reg. }}$ Using lemma 4.5 we get

$$
\left(w-R_{h} w, \theta\right)_{0} \leq c h^{2}\left\|\frac{\partial u}{\partial t}\right\|_{2, \Omega_{1} \cup \Omega_{2}}\|\theta\|_{0} .
$$

Thus we have

$$
\frac{d}{d t}\|\theta\|_{0} \leq c h^{2}\left\|\frac{\partial u}{\partial t}\right\|_{2, \Omega_{1} \cup \Omega_{2}} .
$$


Integration over $[0, t]$ and using $\|\theta(0)\|_{0}=\left\|\hat{u}_{0}-R_{h} u_{0}\right\|_{0}$ proves the desired result.

REMARK 4. We comment on the error analysis for the three-dimensional case. The Nitsche method given in (3.4) has an obvious analogon if we consider a problem as in (1.1)-(1.5) with $\Omega \subset \mathbb{R}^{3}$ and use the extended finite element space on a family of shape regular tetrahedral triangulations. The arguments to derive the consistency result in lemma 4.1 are dimension independent. Results as in lemma 4.2 , lemma 4.4 and lemma 4.5 can be proved using results from [9]. The arguments and the techniques used are essentially the same as for the $2 \mathrm{D}$ case.

5. Time discretization. The semi-discretization (3.4), resulting from Nitsche's method, can be combined with standard time discretization methods. For example, the $\theta$-scheme $(\theta \in(0,1])$ takes the following form. For $n=0,1, \ldots, N-1$, with $N \Delta t=T$, set $u_{h}^{0}:=\hat{u}_{h}^{0} \in V_{h}^{\Gamma}$, and determine $u_{h}^{n+1} \in V_{h}^{\Gamma}$ such that for all $v_{h} \in V_{h}^{\Gamma}$

$$
\left(\frac{u_{h}^{n+1}-u_{h}^{n}}{\Delta t}, v_{h}\right)_{0}+a_{h}\left(\theta u_{h}^{n+1}+(1-\theta) u_{h}^{n}, v_{h}\right)=\left(\theta f\left(t_{n+1}\right)+(1-\theta) f\left(t_{n}\right), v_{h}\right)_{0}
$$

holds. The error analysis of this full discretization method can be performed using standard arguments, as in [26]. For completeness we derive an error bound for the implicit Euler method. Again we require that the solution $u=u(t) \in V_{\text {reg }}$ as defined in lemma 2.1 has sufficient regularity, in particular $\frac{\partial u}{\partial t} \in L^{1}\left(0, T ; W_{\text {reg }}\right)$ and $\frac{\partial^{2} u}{\partial t^{2}} \in$ $L^{1}\left(0, T ; L^{2}(\Omega)\right)$.

ThEOREM 5.1. Let $u=u(t) \in V_{\text {reg }}$ be the solution defined in lemma 2.1 and $u_{h}^{n} \in V_{h}^{\Gamma}, n=0,1, \ldots, N$ the solution of the $\theta$-scheme (5.1) for $\theta=1$. The following holds:

$$
\begin{aligned}
& \left\|u_{h}^{n}-u\left(t_{n}\right)\right\|_{0} \\
& \leq\left\|\hat{u}_{0}-R_{h} u_{0}\right\|_{0}+c h^{2}\left\{\left\|u_{0}\right\|_{2, \Omega_{1} \cup \Omega_{2}}+\int_{0}^{t_{n}}\left\|\frac{\partial u}{\partial t}\right\|_{2, \Omega_{1} \cup \Omega_{2}} d \tau\right\}+\Delta t \int_{0}^{t_{n}}\left\|\frac{\partial^{2} u}{\partial t^{2}}\right\|_{0} d \tau .
\end{aligned}
$$

Proof. We use the splitting $u_{h}^{n}-u\left(t_{n}\right)=\left(u_{h}^{n}-R_{h} u\left(t_{n}\right)\right)+\left(R_{h} u\left(t_{n}\right)-u\left(t_{n}\right)\right)=$ : $\theta^{n}+\rho^{n}$. For $\left\|\rho^{n}\right\|_{0}=\left\|\rho\left(t_{n}\right)\right\|_{0}$ we have a bound as in (4.11). For the backward difference quotient we introduce the notation $\bar{\partial}^{n} w:=\left(w^{n}-w^{n-1}\right) / \Delta t$. Using the definition of $u_{h}^{n}$ in (5.1), the definition of the semi-discretization in (3.4) and the consistency result in lemma 4.1 we obtain

$$
\begin{aligned}
\left(\bar{\partial} \theta^{n}, v_{h}\right)_{0}+a_{h}\left(\theta^{n}, v_{h}\right)= & \frac{1}{\Delta t}\left(u_{h}^{n}-u_{h}^{n-1}, v_{h}\right)_{0}+a_{h}\left(u_{h}^{n}, v_{h}\right) \\
& -\left(\bar{\partial} R_{h} u\left(t_{n}\right), v_{h}\right)_{0}-a_{h}\left(R_{h} u\left(t_{n}\right), v_{h}\right) \\
= & \left(f\left(t_{n}\right), v_{h}\right)_{0}-a_{h}\left(u\left(t_{n}\right), v_{h}\right)-\left(\bar{\partial} R_{h} u\left(t_{n}\right), v_{h}\right)_{0} \\
= & \left(\frac{\partial u\left(t_{n}\right)}{\partial t}, v_{h}\right)_{0}-\left(R_{h} \bar{\partial} u\left(t_{n}\right), v_{h}\right)_{0}=:\left(\omega^{n}, v_{h}\right)_{0},
\end{aligned}
$$

with

$$
\omega^{n}=\frac{\partial u\left(t_{n}\right)}{\partial t}-R_{h} \bar{\partial} u\left(t_{n}\right)=\left[\left(I-R_{h}\right) \bar{\partial} u\left(t_{n}\right)\right]-\left[\bar{\partial} u\left(t_{n}\right)-\frac{\partial u\left(t_{n}\right)}{\partial t}\right]=: \omega_{1}^{n}-\omega_{2}^{n} .
$$

Taking $v_{h}=\theta^{n} \in V_{h}^{\Gamma}$ and using $a_{h}\left(\theta^{n}, \theta^{n}\right) \geq 0$ we get

$$
\left\|\theta^{n}\right\|_{0}^{2}-\left(\theta^{n-1}, \theta^{n}\right) \leq \Delta t\left\|\omega^{n}\right\|_{0}\left\|\theta^{n}\right\|_{0} .
$$


Hence,

$$
\left\|\theta^{n}\right\|_{0} \leq\left\|\theta^{n-1}\right\|_{0}+\Delta t\left\|\omega^{n}\right\|_{0}
$$

and

$$
\left\|\theta^{n}\right\|_{0} \leq\left\|\theta^{0}\right\|_{0}+\Delta t \sum_{j=1}^{n}\left\|\omega^{j}\right\|_{0} \leq\left\|\hat{u}_{0}-R_{h} u_{0}\right\|_{0}+\Delta t \sum_{j=1}^{n}\left\|\omega_{1}^{j}\right\|_{0}+\Delta t \sum_{j=1}^{n}\left\|\omega_{2}^{j}\right\|_{0} .
$$

For $\left\|\omega_{1}^{j}\right\|_{0}$ we obtain with lemma 4.5

$$
\begin{aligned}
\left\|\omega_{1}^{j}\right\|_{0} & =\left\|\frac{1}{\Delta t}\left(I-R_{h}\right) \int_{t_{j-1}}^{t_{j}} \frac{\partial u}{\partial t} d \tau\right\|_{0} \leq \frac{1}{\Delta t} \int_{t_{j-1}}^{t_{j}}\left\|\left(I-R_{h}\right) \frac{\partial u}{\partial t}\right\|_{0} d \tau \\
& \leq c \frac{h^{2}}{\Delta t} \int_{t_{j-1}}^{t_{j}}\left\|\frac{\partial u}{\partial t}\right\|_{2, \Omega_{1} \cup \Omega_{2}} d \tau
\end{aligned}
$$

and thus

$$
\Delta t \sum_{j=1}^{n}\left\|\omega_{1}^{j}\right\|_{0} \leq c h^{2} \int_{0}^{t_{n}}\left\|\frac{\partial u}{\partial t}\right\|_{2, \Omega_{1} \cup \Omega_{2}} d \tau
$$

For $\omega_{2}^{j}$ we have

$$
\Delta t \omega_{2}^{j}=u\left(t_{j}\right)-u\left(t_{j-1}\right)-\Delta t \frac{\partial u\left(t_{j}\right)}{\partial t}=-\int_{t_{j-1}}^{t_{j}}\left(\tau-t_{j-1}\right) \frac{\partial^{2} u(\tau)}{\partial t^{2}} d \tau,
$$

and thus

$$
\Delta t \sum_{j=1}^{n}\left\|\omega_{2}^{j}\right\|_{0} \leq \sum_{j=1}^{n} \int_{t_{j-1}}^{t_{j}}\left(\tau-t_{j-1}\right)\left\|\frac{\partial^{2} u}{\partial t^{2}}\right\|_{0} d \tau \leq \Delta t \int_{0}^{t_{n}}\left\|\frac{\partial^{2} u}{\partial t^{2}}\right\|_{0} d \tau .
$$

Using the results from (5.3), (5.4) in (5.2) in combination with the bound for $\left\|\rho^{n}\right\|_{0}$ from (4.11) we obtain the result.

The analysis of the time discretization given in this section is essentially dimension independent. Key ingredients are sufficient smoothnes of the solution $u$ and the results in lemma 4.2 and lemma 4.5 , cf. remark 4.

REMARK 5. Introducing the transformed variable $\tilde{u}_{h}^{n}:=\beta u_{h}^{n} \in V_{h}^{\Gamma}$ the discretization in (5.1) immediately results in a discretization of the transformed equations (1.6)(1.8), cf. remark 1.

6. Numerical experiments. In this section we present results of numerical experiments. We consider a three-dimensional test problem in which the interface $\Gamma$ is planar. We use this simple interface geometry to avoid errors that are introduced by a numerical interface approximation and to obtain a problem of the form (1.1)-(1.5) with a known and sufficiently smooth solution $u$.

The domain $\Omega=(0,1)^{3}$ is sudivided into the subdomains $\Omega_{1}:=\{(x, y, z) \in$ $\Omega: z<0.34113\}$ and $\Omega_{2}:=\Omega \backslash \Omega_{1}$, which are seperated by the planar interface $\Gamma:=\{(x, y, z) \in \Omega: z=0.34113\}$. The position of the interface and the tetrahedral triangulation (cf. below) are chosen such that these do not fit. 
We consider the problem (1.1)-(1.5) with $\alpha=\left(\alpha_{1}, \alpha_{2}\right):=(1,2), \beta=\left(\beta_{1}, \beta_{2}\right):=$ $(2,1)$ and a stationary velocity field

$$
\mathbf{w}:=(y(1-z), x, 0)^{T},
$$

which satisfies the assumptions (2.1). The right hand side $f$ is taken such that the exact solution is

$$
u(x, y, z, t):= \begin{cases}\exp (-t) \cos (\pi x) \cos (2 \pi y) a z(z+b) & \text { in } \Omega_{1}, \\ \exp (-t) \cos (\pi x) \cos (2 \pi y) z(z-1) & \text { in } \Omega_{2},\end{cases}
$$

where the constants $a$ and $b$ are determined such that the interface conditions (1.2)(1.3) are satisfied. We take homogeneous Dirichlet boundary conditions on the boundary segments $z=0$ and $z=1$ and homogeneous Neumann boundary conditions on the remaining part of the boundary.

6.1. Spatial discretization error bound. For the spatial discretization, we first create a uniform grid with mesh size $h=\frac{1}{N}$, where $N=8,16,32$. Starting from this uniform grid the elements near the interface are refined two times further, i. e. the local mesh size close to the interface is $h_{\Gamma}=\frac{1}{4 N}$. For the case $N=32$ this results in a problem with 1293754 tetrahedra and 226087 unknowns. For the approximation of the initial value we take $\hat{u}_{0}=I_{h}^{*}(u(\cdot, 0))$, with $I_{h}^{*}$ the interpolation operator as in theorem 4.3 .

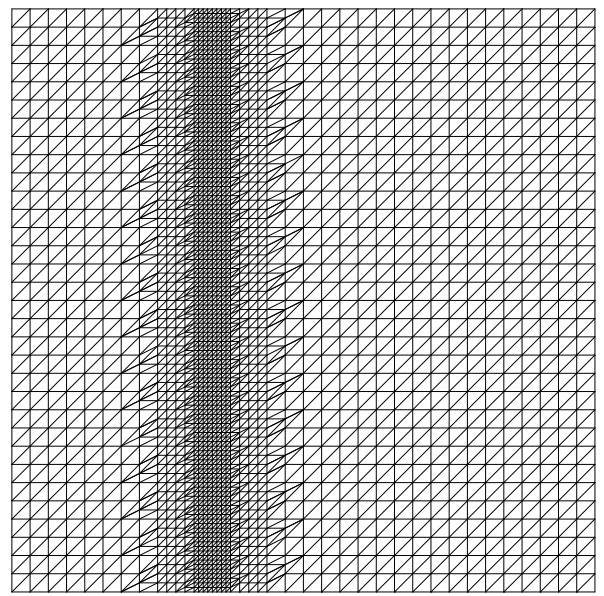

FiG. 6.1. A slice of the tetrahedral mesh at $x=0.25$, for the case $N=16$.

The semi-discretization $u_{h}(t)$ is not known. We computed an accurate approximation of $u_{h}(t)$ using the implicit Euler time-stepping scheme ( (5.1) with $\theta=1$ ) with a time step size $\Delta t$ which is sufficiently small (in our experiments: $\Delta t=10^{-4}$ ) such that the error due to the time discretization is negligible compared to the space discretization error. The resulting reference solution is denoted by $u_{h}^{*}(t)$. In the implementation of (5.1) the basis in $V_{h}^{\Gamma}$ as explained in remark 3 is used. Note that special quadrature methods are needed for computing quantities like $\left(\phi_{i}^{\Gamma}, \phi_{j}\right)_{0}$ and $a_{h}\left(\phi_{i}^{\Gamma}, \phi_{j}\right)$. For the parameter $\lambda$ in the bilinear form $a_{h}(\cdot, \cdot)$ we take the value $\lambda=100$. This choice is based on numerical experiments. It turns out that the error 
behaviour is not very sensitive with respect the choice of the parameter value. The results are essentially the same for all $10^{1} \leq \lambda \leq 10^{3}$.

In Table 6.1 , the errors $\left\|u_{h}^{*}(T)-u(T)\right\|_{L^{2}}$ for $T=0.15$ are displayed. These results are consistent with the theoretical bound $\mathcal{O}\left(h^{2}\right)$ given in theorem 4.6.

\begin{tabular}{|c|c|c|c|}
\hline$N$ & $\left\|u_{h}^{*}(T)-u(T)\right\|_{L^{2}}$ & factor & order \\
\hline 8 & 0.00738506 & - & - \\
16 & 0.00202308 & 3.65 & 1.87 \\
32 & 0.0005228 & 3.87 & 1.95 \\
\hline
\end{tabular}

Spatial discretization error in $L^{2}$-norm and convergence order at $T=0.15$

The exact solution satisfies $[\beta u]_{\Gamma}=0$. In the Nitsche discretization this interface condition is satisfied only approximately. For a stationary symmetric elliptic problem it is shown in [8] that for the discretization $u_{h}$ the error in this interface condition is bounded by $\left\|\left[\beta u_{h}\right]\right\|_{L^{2}(\Gamma)} \leq \operatorname{ch}^{1 \frac{1}{2}}\|u\|_{2, \Omega_{1} \cup \Omega_{2}}$. For the instationary case we were not able to derive a theoretical bound for this error quantity. We computed the errors $\left\|\left[\beta u_{h}^{*}\right]\right\|_{L^{2}(\Gamma)}$ for our problem; the results are given in Table 6.2. It can be observed that the interface condition (1.3) is satisfied only approximately and that the error $\left\|\left[\beta u_{h}^{*}\right]\right\|_{L^{2}(\Gamma)}$ seems to behave like $\mathcal{O}(h)$. The numerical solution for $N=16$

\begin{tabular}{|c|c|c|c|}
\hline$N$ & $\left\|\left[\beta u_{h}^{*}(T)\right]\right\|_{L^{2}(\Gamma)}$ & factor & order \\
\hline 8 & $1.565 e-4$ & - & - \\
16 & $7.975 e-05$ & 1.96 & 0.972 \\
32 & $3.900 e-05$ & 2.05 & 1.03 \\
\hline \multicolumn{4}{|c|}{ TABLE 6.2}
\end{tabular}

$L^{2}$-norm of the jump $\left[\beta u_{h}^{*}(T)\right]_{\Gamma}$ and convergence order at $T=0.15$

at $T=0.15$ in the plane $x=0.25$ is shown in Figure 6.2.

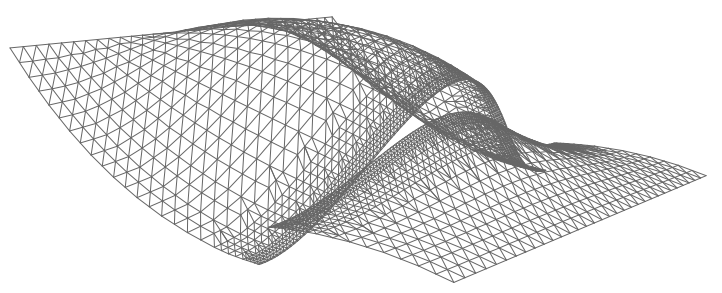

FIG. 6.2. Numerical solution at $T=0.15$ in the plane $x=0.25$. 
6.2. Time discretization error bound. Now we study the time discretization error bound for the implicit Euler method in Theorem 5.1. We use the fixed mesh with $N=16$ as described above and compute a reference solution with $\Delta t=10^{-4}$ in the time interval $[0, T], T=0.2$, which is denoted by $u_{h}^{*}(t)$. The Euler discretization, i.e. (5.1) with $\theta=1$, with time step $\Delta t=\frac{T}{n}$ results in approximations $u_{h}^{n}(T)$ of $u_{h}^{*}(T)$. For the cases $n=5,10,20$ the temporal errors in the $L^{2}$-norm, i.e. $\left\|u_{h}^{n}(T)-u_{h}^{*}(T)\right\|_{L^{2}}$, are given in Table 6.3. We observe the expected first order of convergence in $\Delta t$.

\begin{tabular}{|c|c|c|c|}
\hline$n$ & $\left\|u_{h}^{n}(T)-u_{h}^{*}(T)\right\|_{L^{2}}$ & factor & order \\
\hline 5 & $1.254 e-05$ & - & - \\
10 & $6.092 e-06$ & 2.06 & 1.04 \\
20 & $3.011 e-06$ & 2.02 & 1.02 \\
\hline \multicolumn{4}{|c|}{ TABLE 6.3} \\
\hline
\end{tabular}

Time discretization error in $L^{2}$ norm and convergence order at $T=0.2$

\section{REFERENCES}

[1] T. Belytschko, N. Moes, S. Usui, and C. Parimi, Arbitrary discontinuities in finite elements, Int. J. Num. Meth. Eng., 50 (2001), pp. 993-1013.

[2] D. Bothe, M. Koebe, K. Wielage, J. Prüss, and H.-J. Warnecke, Direct numerical simulation of mass transfer between rising gas bubbles and water, in Bubbly Flows: Analysis, Modelling and Calculation, M. Sommerfeld, ed., Heat and Mass Transfer, Springer, 2004.

[3] D. Bothe, M. Koebe, K. Wielage, and H.-J. Warnecke, VOF-simulations of mass transfer from single bubbles and bubble chains rising in aqueous solutions, in Proceedings 2003 ASME joint U.S.-European Fluids Eng. Conf., Honolulu, 2003, ASME. FEDSM2003-45155.

[4] Z. Chen AND J. Zhou, Finite element methods and their convergence for elliptic and parabolic interface problems, Numer. Math., 79 (1998), pp. 175-202.

[5] J. Chessa and T. Belytschko, An extended finite element method for two-phase fluids, ASME Journal of Applied Mechanics, 70 (2003), pp. 10-17.

[6] A. Ern and J.-L. Guermond, Theory and practice of finite elements, Springer, New York, 2004.

[7] S. Gross, V. Reichelt, And A. Reusken, A finite element based level set method for two-phase incompressible flows, Comp. Visual. Sci., 9 (2006), pp. 239-257.

[8] A. Hansbo And P. Hansbo, An unfitted finite element method, based on nitsche's method, for elliptic interface problems, Comput. Methods Appl. Mech. Engrg., 191 (2002), pp. 55375552 .

[9] — A finite element method for the simulation of strong and weak discontinuities in solid mechanics, Comput. Methods Appl. Mech. Engrg., 193 (2004), pp. 3523-3540.

[10] A. Hansbo, P. Hansbo, and M. Larson, A finite element method on composite grids based on Nitsche's method, Math. Model. Numer. Anal., 37 (2003), pp. 495-514.

[11] P. HansBo, Nitsche's method for interface problems in computational mechanics, GAMMMitt., 28 (2005), pp. 183-206.

[12] B. HeinRICh AND B. Jung, The Fourier-Nitsche-mortaring for elliptic problems with reentrant edges, Computing, 80 (2007), pp. 221-246.

[13] — Nitsche finite element method for parabolic problems, preprint, Department of Mathematics, Technical University Chemnitz, 2008.

[14] B. Heinrich And S. Nicaise, The Nitsche mortar finite-element method for transmission problems with singularities, IMA J. Numer. Anal., 23 (2003), pp. 331-358.

[15] B. HeinRich And K. Pietsch, Nitsche type mortaring for some elliptic problem with corner singularities, Computing, 68 (2002), pp. 217-238.

[16] B. HeINRICH AND K. Pönitz, Nitsche type mortaring for singularly perturbed reaction-diffusion problems, Computing, 75 (2005), pp. 257-279.

[17] M. IshiI, Thermo-Fluid Dynamic Theory of Two-Phase Flow, Eyrolles, Paris, 1975.

[18] J. Lions And E. Magenes, Nonhomogeneous boundary value problems and applications, Springer-Verlag, New York, 1972. 
[19] M. Muradoglu and G. Tryggvason, A front-tracking method for computation of interfacial flows with soluble surfactant, J. Comput. Phys., 227 (2008), pp. 2238-2262.

[20] J. Nitsche, Über ein Variationsprinzip zur Lösung von Dirichlet-Problemen bei Verwendung von Teilräumen, die keinen Randbedingungen unterworfen sind, Abh. Math. Sem. Univ. Hamburg, 36 (1971), pp. 9-15.

[21] A. Reusken, Analysis of an extended pressure finite element space for two-phase incompressible flows, Comp. Visual. Sci., 11 (2008), pp. 293-305.

[22] S. Sadhal, P. Ayyaswamy, and J. Chung, Transport Phenomena with Droplets and Bubbles, Springer, New York, 1997.

[23] J. Slattery, Advanced Transport Phenomena, Cambridge Universtiy Press, Cambridge, 1999.

[24] R. Temam, Infinite-dimensional dynamical systems in mechanics and physics, Springer, New York, 1988.

[25] - Navier-Stokes equations, theory and numerical analysis, North-Holland, Amsterdam, 3rd ed., 1984.

[26] V. ThomeE, Galerkin finite element methods for parabolic problems, Springer, Berlin, 1997.

[27] A.-K. Tornberg And B. Engquist, A finite element based level-set method for multiphase flow applications, Comp. Vis. Sci., 3 (2000), pp. 93-101. 